\title{
Gene expression of benthic amphipods (genus: Diporeia) in relation to a circular ssDNA virus across two Laurentian Great Lakes
}

\author{
Kalia SI Bistolas ${ }^{\text {Corresp., }}{ }^{1}$, Lars G Rudstam ${ }^{2}$, Ian Hewson ${ }^{1}$ \\ 1 Department of Microbiology, Cornell University, Ithaca, New York, United States \\ 2 Department of Natural Resources and the Cornell Biological Field Station, Cornell University, Bridgeport, New York, United States \\ Corresponding Author: Kalia SI Bistolas \\ Email address: ksb97@cornell.edu
}

Circular rep-encoding ssDNA (CRESS-DNA) viruses are common constituents of invertebrate viral consortia. Despite their ubiquity and sequence diversity, the effects of CRESS-DNA viruses on invertebrate biology and ecology remain largely unknown. This study assessed the relationship between the transcriptional profile of benthic amphipods of genus Diporeia and the presence of the CRESS-DNA virus, LM29173, in the Laurentian Great Lakes to provide potential insight into the influence of these viruses on invertebrate gene expression. Twelve transcriptomes derived from Diporeia were compared, representing organisms from two amphipod haplotype clades (Great Lakes Michigan and Superior, defined by COI barcode sequencing) with varying viral loads (up to $3 \times 10^{6}$ genome copies organism ${ }^{-1}$ ). Read recruitment to de novo assembled transcripts revealed 2,208 significantly over or underexpressed contigs in transcriptomes with above average LM29173 load. Of these contigs, 31.5\% were assigned a putative function. The greatest proportion of annotated, differentially expressed transcripts were associated with functions including: (1) replication, recombination and repair, (2) cell structure/biogenesis, and (3) post-translational modification, protein turnover, and chaperones. Contigs putatively associated with innate immunity displayed no consistent pattern of expression, though several transcripts were significantly overexpressed in amphipods with high viral load. Quantitation (RT-qPCR) of target transcripts, non-muscular myosin heavy chain, $\beta$-actin, and ubiquitin-conjugating enzyme E2, corroborated transcriptome analysis and indicated that Lake Michigan and Lake Superior amphipods with high LM29173 load exhibit lakespecific trends in gene expression. While this investigation provides the first comparative survey of the transcriptional profile of invertebrates of variable CRESS-DNA viral load, additional inquiry is required to define the scope of host-specific responses to potential infection. 
Gene expression of benthic amphipods (genus: Diporeia) in relation to a circular ssDNA virus across two Laurentian Great Lakes

Kalia S.I. Bistolas ${ }^{1 *}$, Lars G. Rudstam², Ian Hewson ${ }^{1}$

${ }^{1}$ Department of Microbiology, Cornell University, Ithaca, NY, USA

2 Department of Natural Resources and the Cornell Biological Field Station, Cornell University, Bridgeport, NY, USA

* Corresponding author:

Kalia Bistolas ${ }^{1}$

Department of Microbiology, Wing Hall, 123 Wing Drive, Ithaca, NY 14853, USA. Tel.: +1 607 255 0151. Fax: 607-255-3904.

E-mail address: ksb97@cornell.edu 


\section{Abstract}

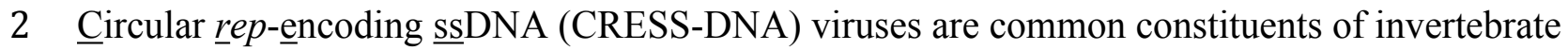

3 viral consortia. Despite their ubiquity and sequence diversity, the effects of CRESS-DNA viruses

4 on invertebrate biology and ecology remain largely unknown. This study assessed the

5 relationship between the transcriptional profile of benthic amphipods of genus Diporeia and the

6 presence of the CRESS-DNA virus, LM29173, in the Laurentian Great Lakes to provide

7 potential insight into the influence of these viruses on invertebrate gene expression. Twelve

8 transcriptomes derived from Diporeia were compared, representing organisms from two

9 amphipod haplotype clades (Great Lakes Michigan and Superior, defined by COI barcode sequencing) with varying viral loads (up to $3 \times 10^{6}$ genome copies organism ${ }^{-1}$ ). Read recruitment

11 to de novo assembled transcripts revealed 2,208 significantly over or underexpressed contigs in 12 transcriptomes with above average LM29173 load. Of these contigs, 31.5\% were assigned a

13 putative function. The greatest proportion of annotated, differentially expressed transcripts were

14 associated with functions including: (1) replication, recombination and repair, (2) cell

15 structure/biogenesis, and (3) post-translational modification, protein turnover, and chaperones.

16 Contigs putatively associated with innate immunity displayed no consistent pattern of

17 expression, though several transcripts were significantly overexpressed in amphipods with high

18 viral load. Quantitation (RT-qPCR) of target transcripts, non-muscular myosin heavy chain, $\beta$ -

19 actin, and ubiquitin-conjugating enzyme E2, corroborated transcriptome analysis and indicated

20 that Lake Michigan and Lake Superior amphipods with high LM29173 load exhibit lake-specific

21 trends in gene expression. While this investigation provides the first comparative survey of the

22 transcriptional profile of invertebrates of variable CRESS-DNA viral load, additional inquiry is

23 required to define the scope of host-specific responses to potential infection. 


\section{Introduction}

25

26

27

Circular $\underline{r} e p$-encoding $\underline{\text { ssDNA }}$ (CRESS-DNA) virus genomes are small $(\sim 1.7-4 \mathrm{~kb})$, circular molecules which encode, at minimum, major open reading frames rep (replication initiator protein) and cap (structural capsid protein; Rosario et al. 2012; Rosario et al. 2015). Eukaryotic CRESS-DNA viruses broadly encompass ssDNA viruses that infect plants (Geminiviridae, Nanoviridae), and metazoans (Circoviridae, Anelloviridae; Dunlap et al. 2013; Rosario et al. 2017; Rosario et al. 2012), and include common and important pathogens of ecologically or commercially relevant vertebrates. For example, beak and feather disease virus (BFDV, Circoviridae) is responsible for persistent immunosuppression in avian hosts (Eastwood et al. 2014) and porcine circoviruses infect domestic swine, manifesting sub-clinically (PCV1) or eliciting postweaning multisystemic wasting syndrome (PMWS, PCV2;Allan \& Ellis 2000). The use of culture-independent (metaviromic) approaches has led to the discovery and characterization of an extraordinary diversity of novel ssDNA viruses in environmental reservoirs and non-model invertebrates (Labonté \& Suttle 2013; Rosario \& Breitbart 2011; Rosario et al. 2017; Rosario et al. 2012; Roux et al. 2016). To date, the etiology, pathology, and association between ssDNA viruses and any invertebrate remains wholly unknown. This study utilized whole transcriptome sequencing to investigate the relationship between a CRESS-DNA virus and benthic amphipods of genus Diporeia from the Laurentian Great Lakes.

CRESS-DNA viruses have been identified in association several major aquatic invertebrate phyla, including the Annelida, Arthropoda, Chaetognatha, Cnidaria, Ctenophora, Echinodermata, and Mollusca, among others (Breitbart et al. 2015; Dayaram et al. 2016; Dunlap et al. 2013; Eaglesham \& Hewson 2013; Fahsbender et al. 2015; Kibenge 2016; Hewson et al. 2013a; Hewson et al. 2013b; Jackson et al. 2016; Rosario et al. 2015; Soffer et al. 2013). These 
47 viruses appear to be biogeographically widespread, taxonomically diverse, and common

48 constituents of crustacean nanobiomes (Dunlap et al. 2013; Hewson et al. 2013a; Hewson et al.

49 2013b; Labonté \& Suttle 2013; Rosario et al. 2017; Rosario et al. 2012; Rosario et al. 2015).

50 However, little is known about the role of CRESS-DNA viruses in mediating crustacean

51 ecology, physiology, and mortality. Because no immortal crustacean cell line currently exists,

52 propagation of crustacean-associated CRESS-DNA viruses in vitro remains intractable, and the

53 unknown nature of CRESS-DNA virus tropism and infection dynamics in these systems impedes

54 targeted sequencing of virus-infected cells. Furthermore, many microcrustaceans cannot be

55 reared or maintained effectively in aquaria without significant physiological stress and high

56 incidence of mortality, hindering in vivo infection experiments. Therefore, we implemented a

57 whole-organism comparative transcriptome sequencing (transcriptomics) approach in evaluating

58 the relationship between the presence of CRESS-DNA viral genotype, LM29173, and benthic

59 crustaceans (genus: Diporeia) in Great Lakes ecosystems.

Diporeia are historically abundant benthic meiofauna in the Laurentian Great Lakes

61 (Auer et al. 2013; Barbiero et al. 2011; Birkett et al. 2015; Guiguer \& Barton 2002). These

62 amphipods influence lake-wide biogeochemistry and mediate relationships between spring

63 diatom blooms and upper trophic level consumers through detritivory and sediment bioturbation

64 (Gardner et al. 1985; Guiguer \& Barton 2002; Halfon et al. 1996; Wells 1980). Localized and

65 precipitous declines in several Diporeia populations have prompted exploration of their viral

66 consortia (Bistolas et al. 2017 ; Hewson et al. 2013a). Metavirome sequencing has documented a

67 common and recurrent CRESS-DNA virus genotype, LM29173, frequently detected in impacted

68 Diporeia populations in Lakes Michigan and Huron, but rare among specimens from stable Lake

69 Superior populations (Bistolas et al. 2017; Hewson et al. 2013a). It is also prevalent among 
70 amphipods from the deep, glacial Finger Lakes of Central New York (Seneca, Cayuga, and

71 Owasco Lakes). Previous DNA barcoding of maternally inherited cytochrome c oxidase I (COI)

72 sequences (Pilgrim et al. 2009; Bistolas et al. 2017) have revealed sub-species genetic variation

73 between impacted and stable populations, with Diporeia from Lakes Michigan, Huron, Ontario,

74 Erie, and the Finger Lakes comprising a southern lake haplotype clade, and amphipods from

75 Lake Superior comprising a northern lake haplotype clade. While LM29173 is more abundant in 76 Diporeia from declining southern populations than stable northern populations, no advances

77 have been made to describe the impact of this CRESS-DNA virus on amphipod biology. This

78 study offers preliminary insight into the relationship between LM29173 and gene expression in

79 amphipods from both haplotype clades, and provides transcriptional targets for further

80 investigation. Specific objectives of this study were to (1) investigate the association between

81 LM29173 presence/load and the transcriptional profile of Diporeia, (2) determine if detected

82 changes in gene expression are specific to distinct Diporeia haplotypes, and (3) explore the 83 effect of LM29173 presence on amphipod transcription of innate immunity regulators/effectors.

85

86

87 88

\section{Materials \& Methods}

Sample collection and transcriptome preparation: Diporeia were collected in August-

September 2014 via Ponar benthic sampler from Great Lakes Michigan and Superior at EPAdesignated stations (Fig. 1, Supplementary Table 1; U.S. Environmental Protection Agency, 2012). Organisms were sieved to remove sediment $(500 \mu \mathrm{m})$, rinsed, and immediately individually frozen at $-80^{\circ} \mathrm{C}$.

Nucleic acids were extracted from individual amphipods via ZR-Duet ${ }^{\mathrm{TM}}$ DNA/RNA MiniPrep kit (Zymo Research, Irvine, CA, USA). Presence and genome load (copy number) of 
93 LM29173 was determined via qPCR per Hewson et al. (2013a) using SsoAdvanced ${ }^{\mathrm{TM}}$ Universal

94 Probes Supermix (Bio-Rad Laboratories, Hercules, CA, USA), corrected for total extraction

95 volume, and standardized by organism wet weight $(\mathrm{mg})$. Two samples with the highest and two

96 samples with the lowest copy numbers organsim ${ }^{-1}$ of LM29173 from each of three stations (Lake

97 Michigan 27 and 40, Lake Superior 066; Fig. 2, Supplementary Fig. 1; U.S. Environmental

98 Protection Agency, 2012) were selected for transcriptome preparation ( $\mathrm{n}=12$ total transcriptomes,

$99 n=4$ per station). For selected samples, RNA fractions were further enzymatically digested with

100 TurboDNAse (Thermo Fisher Scientific, Waltham, MA, USA) for 15min to reduce co-extracted

101 DNA. Ribosomal RNA was depleted via mRNA-ONLYTM mRNA Isolation Kit (Epicentre,

102 Madison, WI, USA), and remaining RNA was reverse transcribed and amplified via the

103 TransPlex ${ }^{\circledR}$ Complete Whole Transcriptome Amplification Kit (WTA2, Sigma-Aldrich, Saint

104 Louis, MO, USA) per manufacturer instructions. Resulting cDNA libraries were quantified via

105 PicoGreen fluorescence and prepared for sequencing using a Nextera XT DNA Library

106 Preparation Kit (Illumina, San Diego, CA, USA). Resulting libraries were subjected to 2x250bp

107 paired-end sequencing on an Illumina MiSeq at the Cornell University Core Laboratories Center

108 (Ithaca, NY, USA). Libraries were deposited in Genbank (accession: PRJNA379017;

109 SRR5341776-SRR5341788).

$110 \quad$ Transcriptome assembly and comparison of transcript expression: Reads were trimmed

111 for quality (quality score $<0.05$, modified-Mott trimming algorithm), ambiguous nucleotides

112 ( $\mathrm{n}=0)$, length $(50 \mathrm{nt} \leq$ length $\leq 251 \mathrm{nt})$, and Illumina adapters via CLC Genomics Workbench

113 (v.8.5.1, Qiagen, Hilden, Germany). Reads mapped to SILVA rRNA databases (90\% identity,

114 50\% coverage via CLC Genomics Workbench; http:/www.arb-silva.de/) were excluded from

115 assembly. Remaining reads were then assembled de novo using Trinity on the Galaxy 
116 bioinformatics platform per default parameters (National Center for Genome Analysis Support,

117 Indiana University Pervasive Technology Institute; Supplementary Table 2). Resulting contigs

118 were further clustered via CD-HIT-EST to reduce isoform redundancy (sequence identity cutoff

$119=0.98$ ). Reads were aligned to contigs via the Bioconductor package EdgeR (Robinson \& Smyth

120 2007) in CLC Genomics Workbench (v.8.5.1, Qiagen, Hilden, Germany) to calculate relative

121 read recruitment (Reads Per Kilobase of transcript per Million mapped reads; RPKM) and

122 significance (corrected for multiple comparison via false discovery rate methods; FDR). Contigs

123 that exhibited $>10$ fold change (EdgeR) in read recruitment, $\Delta \mathrm{RPKM}>100$, and FDR-adjusted

124 p-value $<0.05$ between the six low LM29173 load libraries and six high LM29173 load libraries

125 were considered significantly differentially expressed genes (DEGs). DEGs were then annotated

126 using Blast2Go (v.4.0.7 BLASTx, evalue $<1 \times 10^{-5}$ ) and functionally classified by EuKaryotic

127 Orthologous Group, or "KOG” (Joint Genome Institute).

128 Eight Lake Michigan libraries were grouped by station and viral load to identify DEGs

129 common between both stations in Lake Michigan, minimizing the effect of between-lake genetic

130 and environmental variance. DEGs shared between libraries were defined per the following

131 criteria: $>2$ fold change in expression, $\triangle \mathrm{RPKM}>10$ between libraries, significantly

132 differentially expressed with an FDR-adjusted p-value $<0.05$, and consistently over or

133 underexpressed in both Lake Michigan stations. Contigs fulfilling these criteria were annotated

134 via BLASTx against the non-redundant (nr) database and assessed for relevance to viral infection

135 (Altschul et al. 1990).

136 To identify contigs affiliated with putative immune functions, reference sequences

137 associated with invertebrate innate immunity were collected from the Insect Innate Immunity

138 Database (Brucker et al. 2012) or curated from NCBI protein database queries of keywords in 
139 Table 1 of McTaggart et al., 2009 (keywords listed in Supplementary Table 3). Contigs

140 homologous to these genes were identified via BLASTx (e-value < 1x10-5; Altschul et al. 1990),

141 and the RPKM of those that were $>2$ fold over or underexpressed in both Lake Michigan stations

142 (Mi27 and Mi40) were standardized to total 18s rRNA RPKM per library and depicted via web-

143 based visualization tool, Morpheous (Broad Institute, Cambridge, MA, USA).

$144 \quad$ Quantification (RT-qPCR) of differentially expressed target genes: Whole amphipods

145 were collected in August-September, 2014 at EPA-designated stations (U.S. Environmental

146 Protection Agency, 2012, Supplementary Table 1) in Lakes Michigan, Huron, and Superior and

147 extracted via ZR-Duet ${ }^{\mathrm{TM}}$ DNA/RNA MiniPrep kit (Zymo Research, Irvine, CA, USA). Load of

148 LM29173 was quantified per Hewson et al (2013a). RNA was reverse transcribed (RT) via

149 Superscript III (Invitrogen, Carlsbad, CA, USA per manufacturer instructions). Parallel no-RT

150 controls were generated using identical reaction parameters and no reverse transcriptase. cDNA

151 was subjected to duplex RT-qPCR (quantifying both a gene of interest and a reference gene to

152 control for organism variability) using SsoAdvanced ${ }^{\mathrm{TM}}$ Universal Probes Supermix (Bio-Rad

153 Laboratories, Hercules, CA, USA). Amplicons were gel-purified (Zymoclean ${ }^{\mathrm{TM}}$ Gel DNA

154 Recovery Kit; Zymo Research, Irvine, CA, USA) and cloned (pGEM®-T Easy Vector; Promega,

155 Madison, WI, USA) using JM109 competent E. coli (Invitrogen, Carlsbad, CA, USA). Plasmids

156 were extracted per Zyppy ${ }^{\mathrm{TM}}$ Plasmid Miniprep Kit instructions (Zymo Research, Irvine, CA,

157 USA) and Sanger sequenced (Cornell University Core Laboratories Center, Ithaca, NY, USA) to

158 confirm primer/probe specificity. Reaction parameters and primer, probe, and standard

159 sequences are detailed in Supplementary Table 4.

160 Samples were run in duplicate with congruent duplicate no-RT controls and quantified

161 using duplicate eight-fold standard dilutions (limits of detection described in Supplementary 
162 Table 4). Ct values, quantity, and standard deviation between technical replicates were

163 determined via StepOnePlus software v.2.3 (Foster City, CA, USA). Valid runs were defined by

164 reaction efficiency $>94 \%$ and standard regression linearity $\left(\mathrm{R}^{2}\right)>0.98$. Samples were excluded

165 if Ct standard deviation between replicates was $>0.5$. Quantities were corrected for total

166 extraction and reverse transcription dilutions. Quantities of targets $\beta$-actin (ACT), ubiquitin-

167 conjugating enzyme E2 (UBQ), and non-muscular myosin heavy chain (NMHC) were

168 standardized by copy number of elongation factor-1 $\alpha$ (EF1A) per reaction.

169

\section{Results \& Discussion}

171 Investigation of amphipod transcriptomes revealed differential expression of DNA

172 replication/repair pathways, cytoskeletal architecture, and post-translational modification

173 associated genes in correlation with CRESS-DNA virus load. However, the degree of variability

174 between transcriptomes limited the ability to identify over or underexpression of specific

175 molecular pathways. It is unknown whether vertebrate and invertebrate CRESS-DNA viruses

176 utilize similar pathways of infection, particularly in light of the considerable divergence in

177 sequence homology and genome architecture between groups. Despite this, differentially

178 expressed genes (DEGs) in Diporeia transcriptomes were often homologous to DEGs in porcine

179 circoviral infections, or were associated with putative innate immune functions. Expression of

180 these genes varied between amphipod haplotype clades, suggesting that the transcriptional

181 relationship with LM29173 may have a heritable component. It remains unclear if CRESS-DNA

182 viral load corresponds significantly with ecologically relevant changes in invertebrate

183 physiology. 
Lake Michigan (100\%) than Lakes Huron (66.7\%; Games-Howell, $p<1 \times 10^{-8}$, Ruxton \&

187 Beauchamp 2008) and Superior (30.8\%; Games-Howell, $p<1 \times 10^{-8}$; Ruxton \& Beauchamp

188 2008; Welch's ANOVA, $\mathrm{F}_{2,26.24}=26.4, p=5.21 \times 10^{-7}$ ), congruent with previous observations of

189 the distribution of this genotype (Bistolas et al. 2017). Pilgrim et al (2009) utilized mitochondrial

190 cytochrome c oxidase I (COI) sequences to identify sub-species genetic variation between

191 Diporeia populations among Great Lakes ecosystems, ultimately delineating two clades with

192 distinct haplotype signatures. qPCR results corroborate previous observations that LM29173 is

193 detected in greater abundance in southern lakes haplotype clade populations (Lakes Michigan,

194 Huron, Ontario, Erie, and the Finger Lakes), relative to northern lakes haplotype clade

195 populations (Lake Superior; Bistolas et al. 2017). Because LM29173 was positively detected in

196 all Lake Michigan amphipods, samples with the highest and lowest respective load of LM29173

197 were utilized for transcriptome preparation (Fig. 2, Supplementary Fig. 1).

198

199

Transcriptome assembly and annotation of differentially expressed genes (DEGS):

200 Sequence reads from twelve Diporeia transcriptomes were collated ( $\mathrm{n}=14,702,859$ after

201 trimming and exclusion of rRNA-like sequences) to de novo assemble 82,074 contigs with a

202 mean length of 310nt and N50 value of 290nt (Supplementary Fig. 2, Supplementary Table 2,

203 Supplementary Table 5). Despite rRNA depletion prior to sequencing, computational subtraction

204 of rRNA reads was considerable (0.016-36.95\%), but comparable to previously observed

205 proportions in other studies (Schmieder et al. 2012; Stewart et al. 2010). Less than 1.25\% of all

206 rRNA-mapped reads (90\% identity, 50\% coverage; SILVA rRNA database) were putatively

207 bacterial in origin, indicating that co-infecting microbes may contribute to variation in Diporeia 
208 transcriptional profiles. No transcripts of non-target CRESS-DNA viruses were identified.

209 However, 19 unique contigs (376-6914nt) shared sequence similarity to putative metazoan-

210 associated RNA viruses when compared to a manually curated database of viral RNA-dependent

211 RNA polymerase sequences (GenBank) or the non-redundant database (BLASTx; e-value

$212<1 \times 10-5)$. These contigs were homologous to members of the Nodaviridae $(\mathrm{n}=4)$, Nyamiviridae

$213(\mathrm{n}=1)$, Orthomyxoviridae $(\mathrm{n}=1)$, Peribunyaviridae $(\mathrm{n}=6)$, Phenuiviridae $(\mathrm{n}=2)$, and

214 Rhabdoviridae $(\mathrm{n}=5)$, yet it remains unclear if these sequences represent transient/nonpathogenic

215 viruses or specific pathogens of Diporeia. Furthermore, despite methodological biases favoring

216 amplification of encapsidated RNA viruses, read recruitment to these contigs was negligible

$217\left(5.74 \times 10^{-05}-0.24 \%\right.$ of mapped, non-rRNA reads), indicating that these genotypes may have

218 minimal relative impact on overall amphipod transcription.

219 Due to the lack of a reference Diporeia genome, transcripts were conservatively

220 assembled using an isoform-sensitive algorithm, resulting in a fragmented assembly with

221 multiple isoforms per gene. To reduce redundant read mapping, contigs were grouped into

22259,317 isoform clusters prior to recruitment analysis. Library D130 (Lake Michigan site Mi40;

223 Supplementary Table 5) contained fewer total reads relative to other libraries, but was retained,

224 as relative read recruitment was standardized by sequencing depth per library. The statistical

225 package, EdgeR, detected 2,208 significantly differentially expressed genes (DEGs) between

226 libraries with high and low LM29173 load among three Great Lakes stations (Table 1).

227 Correlative multidimensional scaling (MDS) analyses indicated that transcriptomes do not

228 cluster by viral presence, viral load, station, or haplotype, likely as a result of high variability in

229 ontogeny and life history between organisms (Supplementary Fig. 3). Libraries from Mi27 (Lake

230 Michigan) contained over 7-fold more DEGs than Mi40 (Lake Michigan) or Su066 (Lake 
231 Superior) libraries. 89\% of these transcripts were overexpressed in libraries with high LM29173

232 load (Table 1, Fig. 3) but were small, unannotated, contained no ORFs, and were therefore

233 removed from downstream analyses. Conversely, volcano plots (Fig. 3) illustrate a roughly

234 symmetrical distribution of significantly over and underexpressed genes in libraries from Mi40

235 and Su066 relative to viral load.

236 Due to its evolutionary distance from sequenced model organisms, the Diporeia

237 transcriptome remains incompletely annotated. Therefore, DEGs were broadly annotated by

238 putative function using BLASTx via Blast2Go (v.4.0.7). Successfully identified contigs were

239 further assigned to a euKaryotic Orthologous Group, or "KOG” classification (Joint Genome

240 Institute, Walnut Creek, CA, USA). Contigs that received designations of "general function

241 prediction only" (KOG designation "R") or "function unknown" (KOG designation "S") were

242 excluded from analysis. Among remaining functionally annotated contigs $(n=696)$, most were

243 involved in replication, recombination and repair (KOG designation " $\mathrm{L}$ ", $\mathrm{n}=61$ ), cell

244 wall/membrane/envelope biogenesis (KOG designation “M”, n=32), or post-translational

245 modification, protein turnover, and chaperones (KOG designation "O”, n=26, Fig. 4). These

246 three functions were further investigated for potential relevance to viral infection.

247 DEGs involved in replication, recombination, and repair - KOG " $L$ ”: The proportion of

248 DEGs involved in modulating DNA synthesis and stability may indicate that CRESS-DNA

249 viruses alter or manipulate cellular replication pathways. This is congruent with the dynamics of

250 circoviral infections in vertebrates, which exploit cellular DNA damage responses through a

251 complex kinase cascade, triggering apoptosis and ultimately facilitating viral replication (Wei et

252 al. 2016). Contigs homologous to unclassified DNA binding proteins, DNA modification

253 enzymes, nucleases, histone structural components, and mobile elements/DNA translocases were 
254 differentially expressed. Several DEGs were responsible for chromatin remodeling, indicating a

255 potential correlation between states of nucleosome packaging and viral load. However, many of

256 these transcripts were associated with opposing functions (e.g. DNA methylases and

257 demethylases) and may target different chromatin residues, rendering it difficult to determine if 258 the presence of LM29173 leads to differential transcription.

DEGs involved in cell wall/membrane/envelope biogenesis - KOG "M": Several

260 homologs of cell-surface receptors and transmembrane transporters including cubilin,

261 calsyntenin, choline transporters, and g-protein coupled receptors were differentially expressed

262 in transcriptomes with high LM29173 load. Contigs putatively involved in carapace biogenesis

263 and the production of other structural/connective tissues (keratin, collagen, and elastin), as well

264 as those involved in cell movement and intracellular transport (actin and myosin) were also

265 significantly differentially expressed. These proteins play central roles in cell growth and

266 replication, and differences in their transcription may be an artifact of natural variability between

267 organisms. However, mis-regulation of these proteins is a well-documented response to many

268 metazoan virus infections (Döhner \& Sodeik; Luftig 1982; Yan et al. 2014). For example,

269 cellular entry and trafficking of porcine circoviruses is actin and small GTPase-mediated

270 (Misinzo et al. 2009; Yan et al. 2014). Myosin is also differentially expressed in subclinical

271 PCV-2 infections and may aid in ATP-dependent intracellular transport of viral particles to the

272 nucleus (Arii et al. 2010; Tomás et al. 2009; Vicente-Manzanares et al. 2009; Xiong et al. 2015).

273 DEGs involved in post-translational modification, protein turnover, and chaperones -

$274 K O G$ " $O$ ": Intracellular transporters are commonly exploited by vertebrate-associated CRESS-

275 DNA viruses to facilitate entry into the nucleus (Cao et al. 2014; Misinzo et al. 2009). A

276 transcript homologous to Ran (Ras-family related GTP-binding nuclear protein) was 
277 overexpressed in transcriptomes with moderate and high LM29173 load, and may be implicated

278 in nucleocytoplasmic transport and regulation of cell cycle progression (Avis \& Clarke ; Sazer \&

279 Dasso). Likewise, ubiquitin-conjugating enzyme E2 was overexpressed in libraries with high

280 viral load. This enzyme facilitates covalent attachment of ubiquitin to protein substrates (Liu et

281 al. 2007), and may be exploited by viruses to mis-regulate proteolytic degradation, modify

282 chromatin structure, activate NF- $\kappa \mathrm{B}$ and other innate immune mechanisms, or advance G2/M-

283 phase cells into S-phase (Cheng et al. 2014; Gao \& Luo 2006). For example, PCV2 encodes a

284 protein (ORF3) that co-localizes and interacts with E3 ubiquitin ligase, resulting in upregulation

285 of P53 and induction of apoptotic programs, presumably benefiting viral egress (Liu et al. 2007).

286 Knockdown of ubiquitination conjugating enzymes also stalls cells in the G2/M phase,

287 prohibiting PCV2 from accessing S-phase DNA polymerase necessary for viral propagation

288 (Cheng et al. 2014; Liu et al. 2007).

289

290

$\underline{R T-q P C R}$ supports a haplotype-specific relationship between LM29173 load and

291 amphipod gene expression: Viral load correlated with opposite trends in gene expression

292 (average log-transformed RPKM) between Lake Superior and Lake Michigan transcriptomes in

293 all KOG categories with the exception of "Chromatin structure and dynamics" (B), "Translation,

294 ribosomal structure and biogenesis" (J), and "Energy production and conversion" (C; Fig. 4).

295 Unlike Lake Superior libraries, Lake Michigan libraries with high viral load were associated with

296 elevated average RPKM (Fig. 4). Because gene expression in organisms with high viral load may

297 be predicated on population-specific characteristics, we identified 29 common genes

298 differentially expressed in both Lake Michigan stations Mi27 and Mi40 (shared DEGs).

299 However, only one contig (non-muscular heavy chain myosin; NMHC) was both successfully 
300 annotated and potentially affiliated with viral infection (Fig. 5C,F). Lake-specific transcriptional

301 profiles confound bulk comparison of gene expression in relation to LM29173 load, and density

302 estimation distributions of individual transcripts indicate that intermediate viral load correlates

303 with increased expression in most KOG classes (Supplementary Fig. 4). These patterns could

304 indicate that CRESS-DNA virus presence has no appreciable impact on gene expression.

305 Alternatively, because amphipods from Lakes Michigan and Superior belong to potentially

306 phenotypically distinct clades (Pilgrim et al. 2009), these results may indicate that response to

307 environmental and microbial stressors is haplotype-specific.

308 RT-qPCR quantification of $\beta$-actin (ACT), non-muscular myosin heavy chain (NMHC),

309 and ubiquitin-conjugating enzyme E2 (UBQ) confirmed opposite trends in contig expression in

310 correlation with above average viral load among amphipods from Lake Michigan and Superior

311 (Fig. 5). Contigs DN12114c1g3i8 (230nt), DN12352c3g10i1 (1229nt), and DN135c0g1i1

312 (309nt) exhibited sequence similarity to ACT from penaeid blue shrimp (Litopenaeus

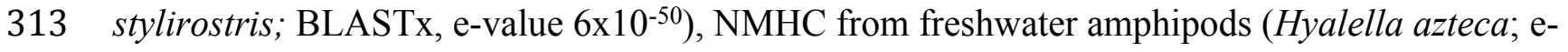

314 value $\left.3.0 \times 10^{-8}\right)$, and UBQ from freshwater amphipods (H. azteca; e-value $\left.4 \times 10^{-52}\right)$, respectively.

315 Relative expression of ACT, NMHC, and UBQ did not significantly correlate with viral load,

316 suggesting that LM29173 does not likely specifically alter transcription of these genes, or the

317 practice of whole-organism RNA extraction obscures cell-specific response(s) to viral presence

318 (Fig. 5). However, relative expression of target genes varied in relation to amphipod population.

319 Organisms associated with the southern haplotype clade exhibited greater average NMHC and

320 UBQ expression, but diminished average ACT expression in concurrence with high viral copy

321 number, relative to amphipods associated with the northern haplotype clade ( $>0.05$, Welch's t-

322 test for all pairwise comparisons). 
324 contig DN11198c0g1i1 (723nt), a homolog of elongation factor 1- $\alpha$ (EF1A) from H. azteca

325 (BLASTx, e-value 2x10-139). This constitutively expressed gene has been validated as an

326 invariant internal RT-qPCR control under experimental conditions in decapods (Leelatanawit et

327 al. 2012), and provided adequate reference to the baseline transcriptional activity of Diporeia, as

328 expression did not correlate with amphipod wet weight, lake, or LM29173 load (Supplementary

329 Fig. 5). Variability in ACT, NMHC, and UBQ expression may be a result of nonspecific RNA

330 extraction, which confounds assessments of specific impacts(s) of viral presence on single tissue

331 types or cells. Additionally, RT-qPCR cannot detect changes in the intracellular localization of

332 myosin subunits nor the state of polymerization of actin subunits, and additional investigation via

333 microscopy and proteomics may be warranted.

336 transcriptomes were surveyed for homologs of genes involved in crustacean innate immunity to

337 determine if LM29173 presence correlates with immune-specific gene expression. 148 homologs

338 (BLASTx e-value $<1 \times 10^{-5}$ ) were identified and exhibited $>2$ fold differential expression in both

339 Lake Michigan station Mi27 and Mi40. Genes involved in stress response (heat shock or

340 oxidative stress response), immune-specific signaling and post-translational modification, and

341 immune-associated cell structure, mobility, and intracellular trafficking mechanisms were

342 consistently overexpressed in Lake Michigan libraries with high viral load (Supplementary Fig.

343 6). Correlative evidence that these immune-related genes are overexpressed in association with

344 high viral load does not preclude the possibility of other co-occurring immune demands. 
345 Therefore, is unclear if overexpression of these genes are a product of environmental stress, or if

346 they are specific responses to viral infection.

347

348 It remains unclear to what extent LM29173 impacts lake-wide Diporeia population

349 dynamics. However, the presence of LM29173 among stable amphipod populations and

350 negligible changes in expression of specific amphipod disease pathways in relation to this viral

351 genotype likely indicate that LM29173 is not solely responsible for Diporeia decline in the

352 Laurentian Great Lakes. We stipulate that CRESS-DNA viruses associated with Diporeia may

353 play a subtle role in altering amphipod physiology, if any. This observation corroborates data

354 from well-characterized mammalian CRESS-DNA viruses (PCV1; Allan \& Ellis 2000; TTV;

355 Okamoto 2009), which often manifest asymptomatically in healthy host tissue. We speculate that

356 LM29173, like other CRESS-DNA viruses, may evade host clearance, attenuate innate immune

357 responses, or elicit host tolerance through post-transcriptional or translational gene regulation,

358 ultimately establishing persistent and asymptompatic infections (Brajão de Oliveira 2015;

359 Okamoto 2009). This hypothesis may explain the universal prevalence and diversity of these

360 viruses in aquatic ecosystems, as observed by metaviromic sequencing.

361

In summary, while LM29173 load does not correlate with significant differential

362 expression of specific gene pathways, transcriptional changes in genes involved in several

363 physiological functions, including innate immunity, are detectable and specific to distinct

364 haplotype clades. To our knowledge, this study communicates the first investigation of the

365 transcriptional relationship between invertebrates and associated CRESS-DNA viruses in natural

366 ecosystems. This study also provides several potential transcriptional targets for further 
367 investigation of gene/pathway-specific inquiries to determine if the bulk of these novel viruses

368 have little effect on metazoan gene expression or physiology.

369

370

\section{Acknowledgements}

We would like to thank Dr. Gary Blissard and Elliot Jackson for indispensable insight into transcriptome analysis and manuscript revision, and Dr. Jim Watkins and the crew of the R/V Guardian (2014) for assistance with sample collection and processing. We also worked closely with the Core Genomics Facility (BRC) at Cornell for sequencing support.

\section{References}

Allan GM, and Ellis JA. 2000. Porcine Circoviruses: A Review. Journal of Veterinary Diagnostic Investigation 12:3-14. 10.1177/104063870001200102

Altschul SF, Gish W, Miller W, Myers EW, and Lipman DJ. 1990. Basic local alignment search tool. Journal of Molecular Biology 215:403-410. 10.1016/s0022-2836(05)80360-2

Arii J, Goto H, Suenaga T, Oyama M, Kozuka-Hata H, Imai T, Minowa A, Akashi H, Arase H, Kawaoka Y, and Kawaguchi Y. 2010. Non-muscle myosin IIA is a functional entry receptor for herpes simplex virus-1. Nature 467:859-862. 10.1038/nature09420 Lake Superior: The Ring of Fire. Journal of Great Lakes Research 39:33-46. 10.1016/j.jglr.2012.12.020

Avis JM, and Clarke PR. Ran, a GTPase involved in nuclear processes: its regulators and effectors. Journal of Cell Science 109:2423-2427. 
383 Barbiero RP, Schmude K, Lesht BM, Riseng CM, Warren GJ, and Tuchman ML. 2011. Trends

384

385

386

387

388

389

390

391

392

393

394

395

396

397

398

399

400

401

402

403

404

in Diporeia populations across the Laurentian Great Lakes, 1997-2009. Journal of Great Lakes Research 37:9-17. 10.1016/j.jglr.2010.11.009

Birkett K, Lozano SJ, and Rudstam LG. 2015. Long-term trends in Lake Ontario's benthic macroinvertebrate community from 1994-2008. Aquatic Ecosystem Health \& Management 18:76-88. 10.1080/14634988.2014.965122

Bistolas KSI, Jackson EW, Watkins JM, Rudstam LG, and Hewson I. 2017. Distribution of circular single-stranded DNA viruses associated with benthic amphipods of genus Diporeia in the Laurentian Great Lakes. Freshwater Biology. 10.1111/fwb.12938

Brajão de Oliveira K. 2015. Torque teno virus: a ubiquitous virus. Revista Brasileira de Hematologia e Hemoterapia 37:357-358. 10.1016/j.bjhh.2015.07.009

Breitbart M, Benner BE, Jernigan PE, Rosario K, Birsa LM, Harbeitner RC, Fulford S, Graham C, Walters A, Goldsmith DB, Berger SA, and Nejstgaard JC. 2015. Discovery, Prevalence, and Persistence of Novel Circular Single-Stranded DNA Viruses in the Ctenophores Mnemiopsis leidyi and Beroe ovata. Frontiers in Microbiology 6:1427. 10.3389/fmicb.2015.01427

Brucker RM, Funkhouser LJ, Setia S, Pauly R, and Bordenstein SR. 2012. Insect Innate Immunity Database (IIID): An Annotation Tool for Identifying Immune Genes in Insect Genomes. PLoS One 7:e45125. 10.1371/journal.pone.0045125

Cao J, Lin C, Wang H, Wang L, Zhou N, Jin Y, Liao M, and Zhou J. 2014. Circovirus Transport Proceeds via Direct Interaction of the Cytoplasmic Dynein IC1 Subunit with the Viral Capsid Protein. Journal of Virology 89:2777-2791. 10.1128/jvi.03117-14 
405 Cheng S, Yan W, Gu W, and He Q. 2014. The ubiquitin-proteasome system is required for the 406 early stages of porcine circovirus type 2 replication. Virology 456-457:198-204.

407

408

409

410

411

412

413

414

415

416

417

418

419

420

421

422

423

424

425

426

427 10.1016/j.virol.2014.03.028

Dayaram A, Galatowitsch ML, Argüello-Astorga GR, van Bysterveldt K, Kraberger S, Stainton D, Harding JS, Roumagnac P, Martin DP, Lefeuvre P, and Varsani A. 2016. Diverse circular replication-associated protein encoding viruses circulating in invertebrates within a lake ecosystem. Infection, Genetics and Evolution 39:304-316. 10.1016/j.meegid.2016.02.011

Döhner K, and Sodeik B. The Role of the Cytoskeleton During Viral Infection. Current Topics in Microbiology and Immunology, 285:67-108.

Dunlap DS, Ng TFF, Rosario K, Barbosa JG, Greco AM, Breitbart M, and Hewson I. 2013. Molecular and microscopic evidence of viruses in marine copepods. Proceedings of the National Academy of Sciences 110:1375-1380. 10.1073/pnas.1216595110

Eaglesham JB, and Hewson I. 2013. Widespread detection of circular replication initiator protein (rep)-encoding ssDNA viral genomes in estuarine, coastal and open ocean net plankton. Marine Ecology Progress Series 494:65-72. 10.3354/meps10575

Eastwood JR, Berg ML, Ribot RFH, Raidal SR, Buchanan KL, Walder KR, and Bennett ATD. 2014. Phylogenetic analysis of beak and feather disease virus across a host ring-species complex. Proceedings of the National Academy of Sciences 111:14153-14158. 10.1073/pnas.1403255111

Fahsbender E, Hewson I, Rosario K, Tuttle AD, Varsani A, and Breitbart M. 2015. Discovery of a novel circular DNA virus in the Forbes sea star, Asterias forbesi. Arch Virol 160:23492351. $10.1007 / \mathrm{s} 00705-015-2503-2$ 
428 Frederick S. B. Kibenge MG. 2016. Aquaculture Virology. London, UK: Academic Press, $429 \quad$ Elsevier.

430 Gao G, and Luo H. 2006. The ubiquitin-proteasome pathway in viral infections. Canadian 431 Journal of Physiology and Pharmacology 84:5-14. 10.1139/y05-144

432 Gardner WS, Nalepa TF, Frez WA, Cichocki EA, and Landrum PF. 1985. Seasonal Patterns in 433 Lipid Content of Lake Michigan Macroinvertebrates. Canadian Journal of Fisheries and Aquatic Sciences 42:1827-1832. 10.1139/f85-229

435

436

437

438

439

440

441

442

443

444

445

446

447

448

449

Guiguer KRRA, and Barton DR. 2002. The Trophic Role of Diporeia (Amphipoda) in Colpoys Bay (Georgian Bay) Benthic Food Web: A Stable Isotope Approach. Journal of Great Lakes Research 28:228-239. 10.1016/s0380-1330(02)70579-0

Halfon E, Schito N, and Ulanowicz RE. 1996. Energy flow through the Lake Ontario food web: conceptual model and an attempt at mass balance. Ecological Modelling 86:1-36. 10.1016/0304-3800(94)00195-2

Hewson I, Eaglesham JB, Höök TO, LaBarre BA, Sepúlveda MS, Thompson PD, Watkins JM, and Rudstam LG. 2013a. Investigation of viruses in Diporeia spp. from the Laurentian Great Lakes and Owasco Lake as potential stressors of declining populations. Journal of Great Lakes Research 39:499-506. 10.1016/j.jglr.2013.06.006

Hewson I, Ng G, Li W, LaBarre BA, Aguirre I, Barbosa JG, Breitbart M, Greco AW, Kearns CM, Looi A, Schaffner LR, Thompson PD, and Hairston NG. 2013b. Metagenomic identification, seasonal dynamics, and potential transmission mechanisms of a Daphniaassociated single-stranded DNA virus in two temperate lakes. Limnology and Oceanography 58:1605-1620. 10.4319/10.2013.58.5.1605 
450 Jackson EW, Bistolas KS, Button JB, and Hewson I. 2016. Novel Circular Single-Stranded DNA

451 Viruses among an Asteroid, Echinoid and Holothurian (Phylum: Echinodermata). PLoS

$452 \quad$ One 11:e0166093. 10.1371/journal.pone.0166093

453 Labonté JM, and Suttle CA. 2013. Previously unknown and highly divergent ssDNA viruses

$454 \quad$ populate the oceans. The ISME Journal 7:2169-2177. 10.1038/ismej.2013.110

455 Leelatanawit R, Klanchui A, Uawisetwathana U, and Karoonuthaisiri N. 2012. Validation of

456 Reference Genes for Real-Time PCR of Reproductive System in the Black Tiger Shrimp.

$457 \quad$ PLoS One 7:e52677. 10.1371/journal.pone.0052677

458 Liu J, Zhu Y, Chen I, Lau J, He F, Lau A, Wang Z, Karuppannan AK, and Kwang J. 2007. The

459

ORF3 Protein of Porcine Circovirus Type 2 Interacts with Porcine Ubiquitin E3 Ligase

460

461

Pirh2 and Facilitates p53 Expression in Viral Infection. Journal of Virology 81:9560-

462

463

9567. 10.1128/jvi.00681-07

Luftig RB. 1982. Does the cytoskeleton play a significant role in animal virus replication?

463

Journal of Theoretical Biology 99:173-191. 10.1016/0022-5193(82)90397-6

464 Misinzo G, Delputte PL, Lefebvre DJ, and Nauwynck HJ. 2009a. Porcine circovirus 2 infection

465 of epithelial cells is clathrin-, caveolae- and dynamin-independent, actin and Rho-

466

467

GTPase-mediated, and enhanced by cholesterol depletion. Virus Research 139:1-9.

468 Okamoto H. 2009. History of Discoveries and Pathogenicity of TT Viruses. Current Topics in $469 \quad$ Microbiology and Immunology 331:1-20

470 Pilgrim EM, Scharold JV, Darling JA, and Kelly JR. 2009. Genetic structure of the benthic 471 amphipod Diporeia (Amphipoda: Pontoporeiidae) and its relationship to abundance in 
474 Robinson MD, and Smyth GK. 2007. Small-sample estimation of negative binomial dispersion, 475 with applications to SAGE data. Biostatistics 9:321-332. 10.1093/biostatistics/kxm030

476 Rosario K, and Breitbart M. 2011. Exploring the viral world through metagenomics. Current Opinion in Virology 1:289-297. 10.1016/j.coviro.2011.06.004

478

479

480

481

482

483

484

485

486

487

488

489

490

491

492

493

Rosario K, Breitbart M, Harrach B, Segalés J, Delwart E, Biagini P, and Varsani A. 2017.

Revisiting the taxonomy of the family Circoviridae: establishment of the genus Cyclovirus and removal of the genus Gyrovirus. Archives of Virology 162:1447-1463. $10.1007 / \mathrm{s} 00705-017-3247-\mathrm{y}$

Rosario K, Duffy S, and Breitbart M. 2012. A field guide to eukaryotic circular single-stranded DNA viruses: insights gained from metagenomics. Archives of Virology 157:1851-1871. 10.1007/s00705-012-1391-y

Rosario K, Schenck RO, Harbeitner RC, Lawler SN, and Breitbart M. 2015. Novel circular single-stranded DNA viruses identified in marine invertebrates reveal high sequence diversity and consistent predicted intrinsic disorder patterns within putative structural proteins. Frontiers in Microbiology 6:696. 10.3389/fmicb.2015.00696

Roux S, Solonenko NE, Dang VT, Poulos BT, Schwenck SM, Goldsmith DB, Coleman ML, Breitbart M, and Sullivan MB. 2016. Towards quantitative viromics for both doublestranded and single-stranded DNA viruses. PeerJ 4:e2777. 10.7717/peerj.2777

Ruxton GD, and Beauchamp G. 2008. Time for some a priori thinking about post hoc testing. Behavioral Ecology 19:690-693. 10.1093/beheco/arn020 
494 Sazer S, and Dasso M. 2000. The ran decathlon: multiple roles of Ran. Journal of Cell Science, $495 \quad 113: 1111-1118$.

496 Schmieder R, Lim YW, and Edwards R. 2012. Identification and removal of ribosomal RNA 497 sequences from metatranscriptomes. Bioinformatics 28:433-435.

498 10.1093/bioinformatics/btr669

499

500 Soffer N, Brandt ME, Correa AMS, Smith TB, and Thurber RV. 2013. Potential role of viruses

501 in white plague coral disease. The ISME Journal 8:271-283. 10.1038/ismej.2013.137

502 Steward FJ, Ottesen EA, and DeLong EF. 2010. Development and quantitative analyses of a 503 universal rRNA-subtraction protocol for microbial metatranscriptomics. The ISME

504 Journal 4:896-907. 10.1038/ismej.2010.18

505 Tomás A, Fernandes LT, Sánchez A, and Segalés J. 2009. Time course differential gene 506

507 expression in response to porcine circovirus type 2 subclinical infection. Veterinary Research 41:12. 10.1051/vetres/2009060

508 United States Environmental Protection Agency. 2012. Quality Assurance Project Plan for the 509 Great Lakes Water Quality Surveys 2008- 2012. Washington, D.C: United States Environmental Protection Agency, Office of Water, Office of Wetlands, Oceans and

512 Vicente-Manzanares M, Ma X, Adelstein RS, and Horwitz AR. 2009. Non-muscle myosin II 513 514 takes centre stage in cell adhesion and migration. Nature Reviews Molecular Cell Biology $10: 778-790.10 .1038 / \mathrm{nrm} 2786$

515 Wei L, Zhu S, Wang J, Quan R, Yan X, Li Z, Hou L, Wang N, Yang Y, Jiang H, and Liu J. 516 2016. Induction of a Cellular DNA Damage Response by Porcine Circovirus Type 2 
Facilitates Viral Replication and Mediates Apoptotic Responses. Scientific Reports 6.

$518 \quad 10.1038 /$ srep39444

519 Wells L. 1980. Food of alewives, yellow perch, spottail shiners, trout-perch, and slimy and 520 fourhorn sculpins in southeastern Lake Michigan.

521 Xiong D, Du Y, Wang HB, Zhao B, Zhang H, Li Y, Hu LJ, Cao J-Y, Zhong Q, Liu WL, Li MZ, 522 Zhu XF, Tsao SW, Hutt-Fletcher LM, Song E, Zeng YX, Kieff E, and Zeng MS. 2015.

523 Nonmuscle myosin heavy chain IIA mediates Epstein-Barr virus infection of

524 nasopharyngeal epithelial cells. Proceedings of the National Academy of Sciences

$525 \quad$ 112:11036-11041. 10.1073/pnas.1513359112

526 Yan M, Zhu L, and Yang Q. 2014. Infection of Porcine Circovirus 2 (PCV2) in Intestinal Porcine 527 Epithelial Cell Line (IPEC-J2) and Interaction between PCV2 and IPEC-J2

528 Microfilaments. Virology Journal 11. 10.1186/s12985-014-0193-0 
Figure 1

Amphipod collection sites in the Laurentian Great Lakes (August - September, 2014).

Collection locations are congruent with EPA-Great Lakes National Program Office (GLNPO) designated stations (U.S. Environmental Protection Agency, 2012). Specimens were collected on the R/V Lake Guardian via Ponar benthic sampler. Bathymetry dataset was provided by NOAA National Geophysical Data Center's Marine Geology \& Geophysics Division (NGDC/MGG) and the NOAA Great Lakes Environmental Research Laboratory (GLERL). Map service published and hosted by Esri Canada ๑ 2012 under Attribution-NonCommercial 2.5 Canada (CC BY-NC 2.5 CA) license https://creativecommons.org/licenses/by-nc/2.5/ca/.

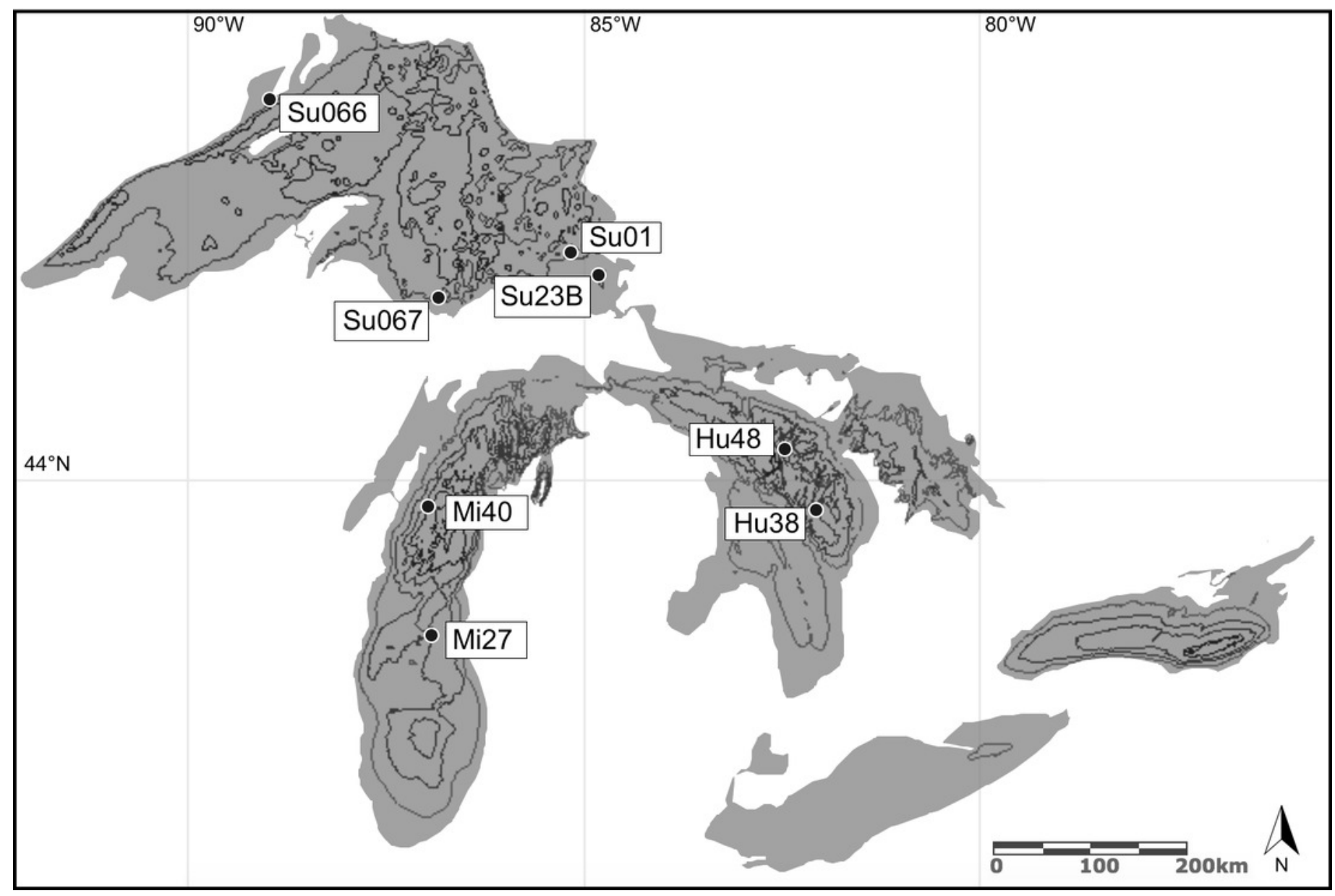




\section{Figure 2}

Quantitative detection of LM29173.

(A) Prevalence and average load ( $\log _{10}$ transformed copy number $\mathrm{mg}^{-1}$ of tissue $\pm 1 \mathrm{SE}$ ) of CRESS-DNA virus genotype LM29173 in amphipods from Great Lakes Michigan, Huron, and Superior. Viral load was significantly greater in Lake Michigan than Lakes Huron (GamesHowell post hoc $t=7.30, p=3.1 \times 10^{-9}$ ) or Superior (Games-Howell post hoc $t=7.30, p=$ 3.0x10-9); (B) Load of LM29173 (copy number organism ${ }^{-1}$ ) in amphipods selected for transcriptome sequencing. Four samples (two with the highest and two with the lowest viral load) were selected from each of three stations: Lake Michigan 27 (Mi27), Lake Michigan 40 (Mi40), and Lake Superior 066 (Su066). Individual transcriptomes are denoted by sample ID $(\# 71,72,75,77,121,128,139,130,358,359,361$, and 362$)$.
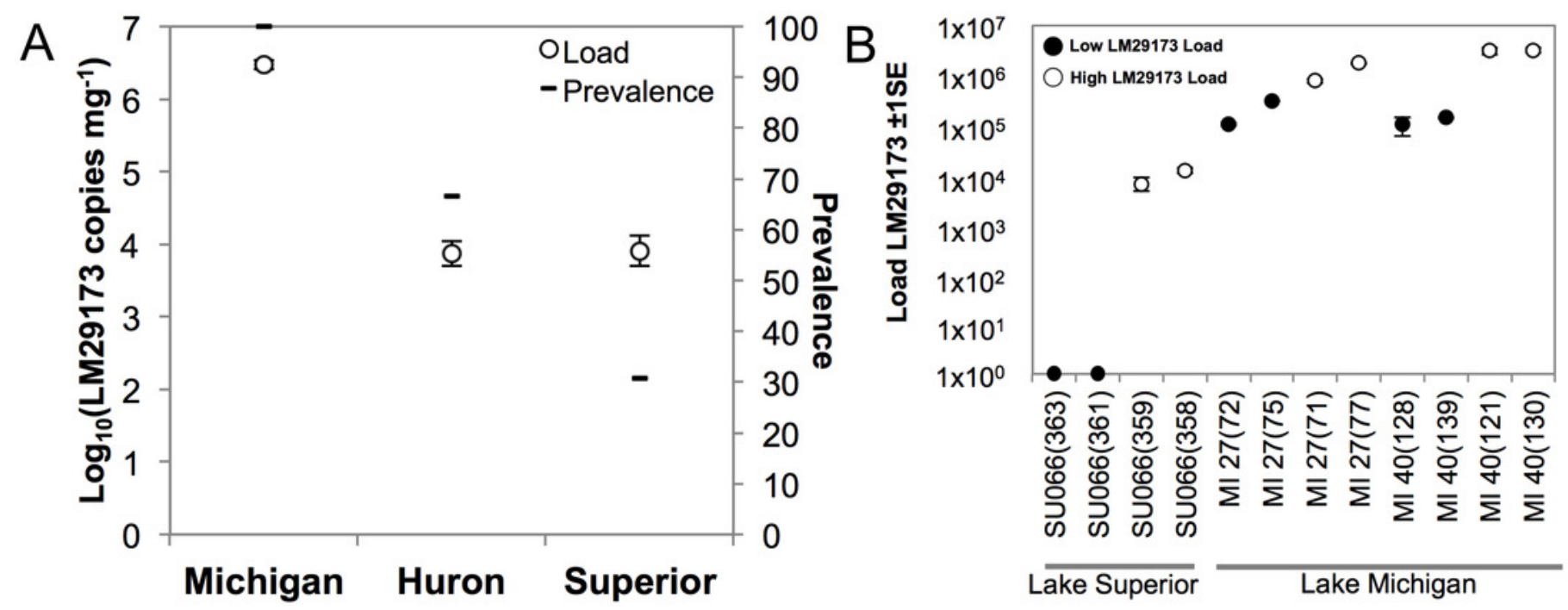


\section{Figure 3}

Volcano plots depicting the distribution of differentially expressed contigs.

Distribution of differentially expressed contigs was determined by EdgeR (Robinson and Smyth, 2008) for libraries from each of three stations: Superior 066 (Su066), Michigan 40 (Mi40), and Michigan 27 (Mi27). Orange points indicate $>10$ fold differentially expressed contigs ( $x$-axis, as determined via EdgeR); red points indicate significantly differentially expressed contigs ( $y$-axis, FDR-adjusted $p$-value $<0.05$ ).
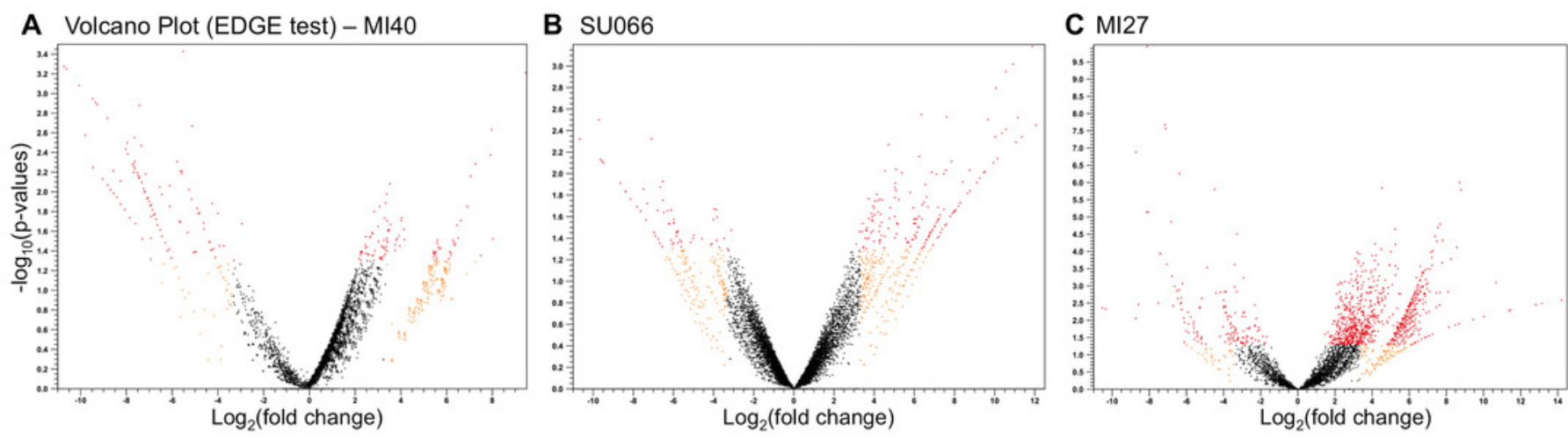


\section{Figure 4}

Average amphipod transcript expression in relation to LM29173 load.

Average expression $\left(\log _{10}(\mathrm{RPKM}+1)\right)$ of contigs in transcriptomes associated with above (grey) and below (white) average LM29173 load. Arrows indicate greater $(\uparrow)$ or reduced $(\downarrow)$ average transformed RPKM in transcriptomes with high LM29173 load relative to transcriptomes with low LM29173 load. Contigs are grouped by putative functional annotation (KOG, Eu K aryotic O rthologous $\mathrm{G}$ roups), and abbreviations correspond to the following functions: (B) chromatin structure and dynamics, $(C)$ energy production and conversion, (D) cell cycle control, cell division, chromosome partitioning, (F) nucleotide transport and metabolism, (I) lipid transport and metabolism, (J) translation, ribosomal structure and biogenesis, $(\mathrm{K})$ transcription, (L) replication, recombination and repair, (M) cell wall/membrane/envelope biogenesis, $(\mathrm{N})$ cell motility, $(\mathrm{O})$ posttranslational modification, protein turnover, chaperones, $(\mathrm{T})$ signal transduction mechanisms, (U) intracellular trafficking, secretion, and vesicular transport, (V) defense mechanisms, (W) extracellular structures. 


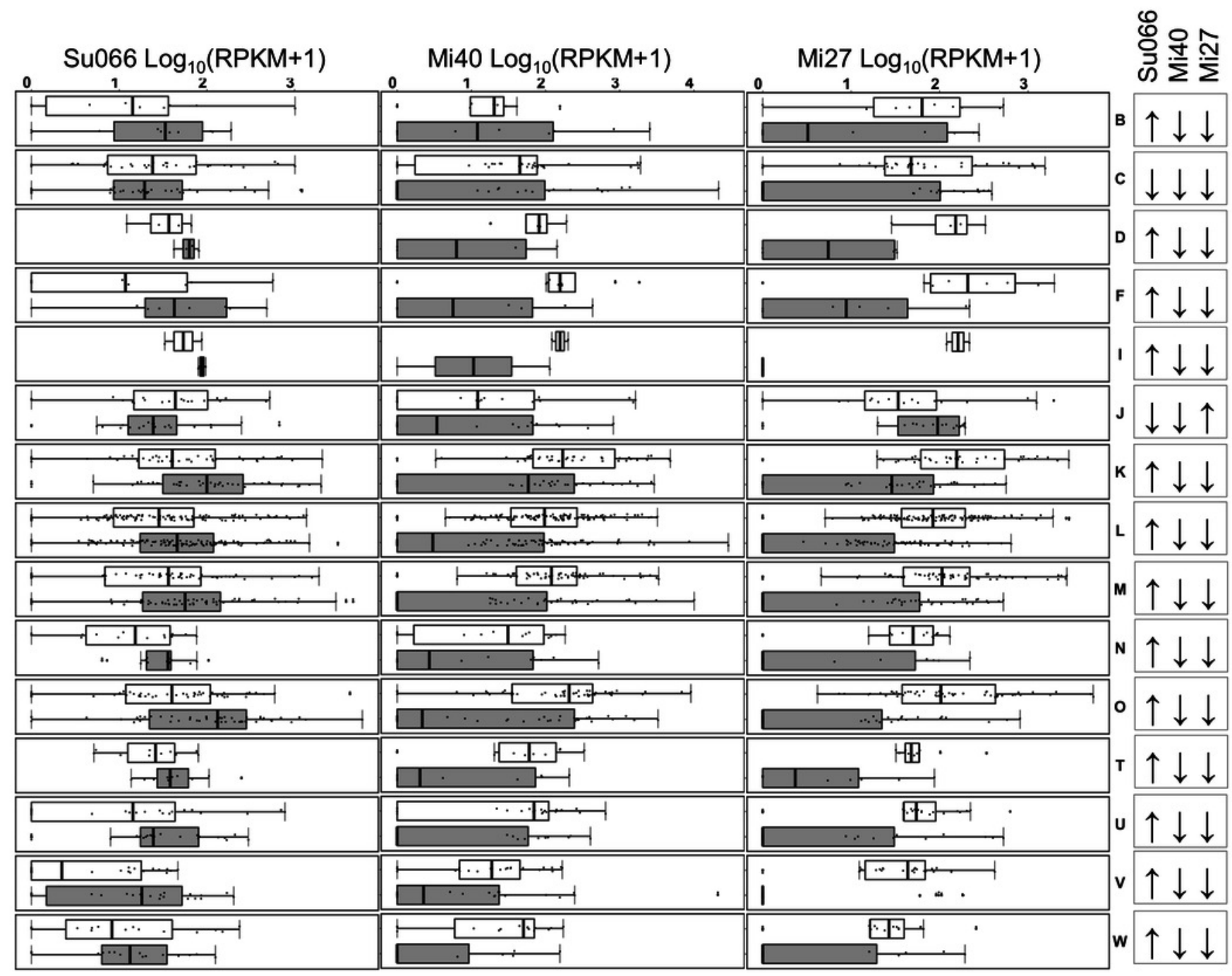




\section{Figure 5}

Relative expression of target genes ACT, UBQ, and NMHC in relation to LM29173 load.

(A-C) Relative expression of target genes $\beta$-actin $(A ; A C T)$, ubiquitin-conjugating enzyme E2 (B; UBQ) and non-muscular myosin heavy chain (C; NMHC) in relation to expression of reference gene elongation factor- $1 \alpha(E F 1 A)$ in specimens from two haplotype clusters (Northern, Southern) with above (grey) and below (white) average LM29173 copy number ( $\pm 1 \mathrm{SE}$ ). (D-F) Correlation between viral load and relative expression of ACT (D), UBQ (E), and NMHC (F) in relation to EF1A reference gene expression. Quantities of target amplicons were standardized by reference gene EF1A using the following equation: (Target ${ }_{\mathrm{RT}}-$ Target $\left._{\mathrm{NRT}}\right) /$ $\left(E F 1 A_{R T}-E F 1 A_{N R T}\right)$, where RT and NRT indicate samples that have been reverse transcribed via Superscript III (Invitrogen, Carlsbad, CA, USA), or not reverse transcribed (no-RT control), respectively. (G) Gene expression (Reads Per Kilobase of transcript per Million mapped reads; RPKM) of target genes $\beta$-actin (ACT), nonmuscular myosin heavy chain (NMHC), and ubiquitin-conjugating enzyme E2 (UBQ) per transcriptome library in each of three stations: Lake Superior station 066 (SU066), Lake Michigan station 40 (Mi40) and Lake Michigan station 27 (Mi27). Libraries are ranked from left to right by increasing LM29173 load. 

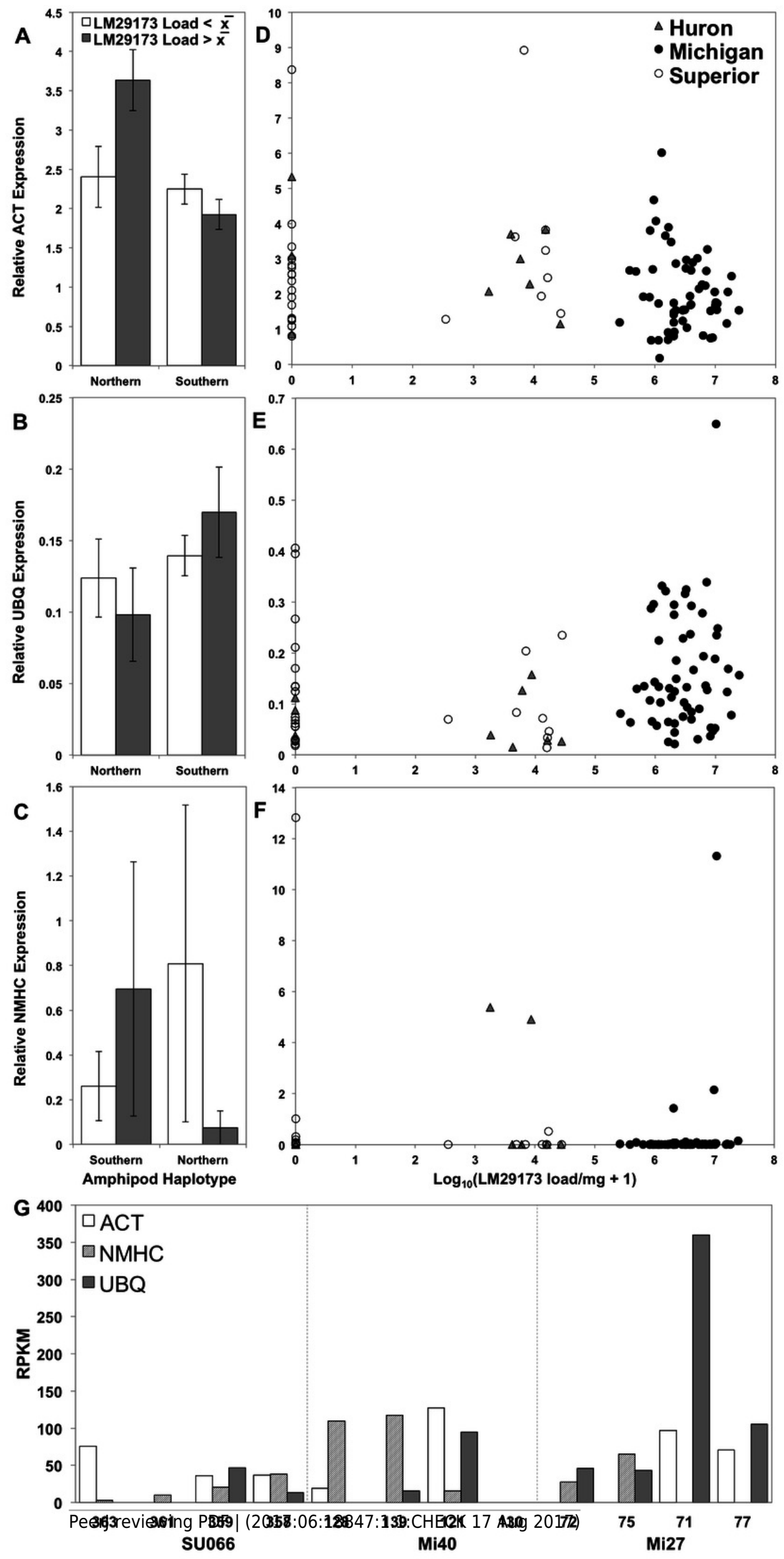


\section{Table $\mathbf{1}$ (on next page)}

Total number of over- and underexpressed contigs in transcriptomes with above average LM29173 load.

Contigs that exhibited $>10$ fold change (EdgeR), $\triangle R$ PKM $>100$, and FDR-adjusted $p$-value $<$ 0.05 were considered significantly differentially expressed genes (DEGs). 
1

\begin{tabular}{|c|c|c|c|}
\hline Library & $\begin{array}{c}\text { Over- } \\
\text { expressed }\end{array}$ & $\begin{array}{c}\text { Under- } \\
\text { expressed }\end{array}$ & Total \\
\hline SU066 & 169 & 65 & 234 \\
\hline MI40 & 129 & 161 & 290 \\
\hline MI27 & 1497 & 187 & 1684 \\
\hline
\end{tabular}

2 